\title{
Effect of Surface Treatments on the Flexural Strength of Heat- Pressed Zirconia Reinforced Lithium Silicate Ceramic and Heat- Pressed Lithium Disilicate Ceramic
}

\section{Hakim $A^{1 *}$, Papathanassiou $A^{2}$, Ali $A^{2}$, Finkelman $\mathbf{M}^{3}$ and Kostagianni $A^{2}$}

${ }^{1}$ Graduate, Department of Prosthodontics, Tufts University School of Dental Medicine, USA

${ }^{2}$ Department of Prosthodontics, Tufts University School of Dental Medicine, Boston, MA, USA

${ }^{3}$ Department of Public Health and Community Service, Tufts University School of Dental

Medicine, USA

*Corresponding author: Adnan Hakim, Graduate, Advanced Education in Esthetic and Operative Dentistry (AEEOD) program, Department of Prosthodontics, Tufts University School of Dental Medicine, Boston, MA, USA, Email: dr.hakim.dent@hotmail.com

\section{Abstract}

Objective: To compare the flexural strength of heat-pressed zirconia-reinforced lithium silicate (Celta Press) with that of heatpressed lithium disilicate (IPS e.max Press) and to evaluate the effect of surface treatments.

Materials and Methods: Sixty-four specimens, with dimensions of 14.5 x $12.5 \mathrm{~mm}$ and $1.5 \mathrm{~mm}$ thickness, were fabricated using the lost-wax heat press technique and were divided into two groups: Group 1, Celta Press and Group 2, IPS e.max Press. Each group had four subgroups according to the surface treatment protocols: No treatment (A); Polished (B); Glazed (C), and Polished and Glazed (D). The specimens' flexural strength was calculated by a universal testing machine in MPa.

Results: IPS e.max Group D, showed the greatest flexural strength (374.22 \pm 87.35MPa), while Celtra Press Group C, had the lowest flexural value $(164.10 \pm 51.97 \mathrm{MPa})$. A two-way ANOVA indicated that IPS e.max exhibited statistically significantly greater flexural strength than Celtra Press. Tukey's HSD test revealed that the polished and glazed group displayed greater flexural strength than the untreated group.

Conclusion: IPS e.max Press showed greater flexural strength than Celtra Press among all groups. The combination of a polished and glazed surface produced greater flexural strength than the untreated group.

Clinical Significance: With newer dental ceramic materials constantly emerging, there is a need to evaluate their mechanical properties and provide clinical recommendations. The aim of this in vitro study is to compare the flexural strength of two highly esthetic ceramic materials, Celtra Press and IPS e.max Press, and to offer some guidelines regarding their surface treatment protocol.

Keywords: Dental ceramics; Surface treatment; Flexural strength; Zr02; Lithium disilicate 


\section{Open Access Journal of Dental Sciences}

\section{Introduction}

All ceramic materials traditionally used, such as feldspathic porcelain, consist of a high glass content that provides increased translucency, but lowers their resistance to high masticatory forces [1]. Conversely, a polycrystalline phase was incorporated that resulted in restorations with greater flexural strength, however it was also associated with more opaque optical properties [2,3]. Lithium disilicate (IPS e.max Press, Ivoclar Vivadent, Schaan, Liechtenstein) is a particle-filled glass ceramic system that has demonstrated both good optical properties and enhanced flexural strength. These mechanical properties have permitted the fabrication of bi-layered anterior crowns, as well as monolithic posterior restorations $[4,5]$. Two different fabrication methods are currently available: Heat pressing and Computer-AidedDesign/Computer-Aided-Manufacturing (CAD/CAM) technique. IPS e.max Press consists of 3 to $6 \mu \mathrm{m}$-long, needle-like crystals of lithium disilicate in a volume fraction of approximately $70 \%$, compared to the rod-like crystals in the sintered CAD/CAM material. These elongated crystals give the pressable ceramic a slightly better flexural strength, approximately 400MPa [6].

Recently, in an effort to create a stronger, but still highly esthetic ceramic material, zirconia-reinforced lithium silicate (Celtra Press, Dentsply Sirona, York, PA) was developed, which claims to combine the superior mechanical properties of yttria-stabilized zirconia (Y-TZP) with the translucency of lithium disilicate. Specifically, it contains $10 \%$ zirconium dioxide $\left(\mathrm{ZrO}_{2}\right)$ and its crystallites are four to eight times smaller than the crystals of lithium disilicate. Moreover, it has a lower percentage of crystal phase content (40$50 \%$ ), and can be finished by both glaze-firing and surface polishing [7]. The final outcome is meant to have a very fine microstructure that combines increased flexural strength with high translucency.

Ceramics' surface microstructure may affect the restoration's esthetic outcome, fracture resistance, and overall longevity [8-10]. Numerous studies have examined surface treatments' effects on ceramic restorations and agreed that polishing and glazing have superior results [1114]. However, there is insufficient data comparing the effect of surface treatments on zirconia-reinforced lithium silicate ceramics and lithium disilicate ceramics.

Hence, the purpose of this in-vitro study was to compare the flexural strength of heat-pressed zirconia-reinforced lithium silicate with that of lithium disilicate ceramic and evaluate the surface treatments in terms of their fracture resistance. The hypothesis was that Celtra Press's flexural strength would exceed that of IPS e.max Press and that the combination of a glazed and polished surface treatment would achieve greater flexural strength than all others.

\section{Material and Methods}

The program nQuery Advisor (Version 7.0) was used to conduct a sample size calculation. Based on the effect size found in a pilot study, a sample size of $n=8$ per subgroup was adequate to obtain a power greater than $99 \%$ to detect a difference between the materials, as well as a power greater than $99 \%$ to detect a difference between the surface treatments, using a significance level of $\alpha=5 \%$. The pilot study had a sample size of $n=3$ per subgroup.

Sixty-four specimens of heat-pressed ceramic discs, with dimensions of $14.5 \times 12.5 \mathrm{~mm}$ and $1.5 \mathrm{~mm}$ thickness, were fabricated using a lost-wax heat press technique according to the manufacturer's instructions. The specimens were divided into two groups: Group (1), zirconia-reinforced lithium silicate ZLS (Celtra Press, MT ingots, A2 Shade; Dentsply Sirona, York, PA) and Group (2), lithium disilicate ceramics (IPS e.max Press, MT ingots, A2 Shade; IvoclarVivadent, Schaan, Liechtenstein). The specimens were then divided into four subgroups according to the surface treatment protocol: No Treatment (A); Polished (B); Glazed (C) and Polished and Glazed (D) (Figure 1).

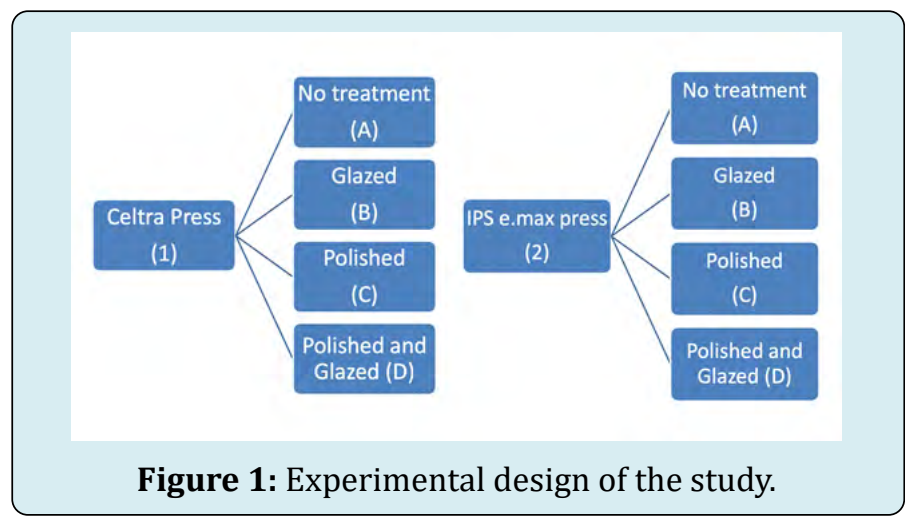

A $14.5 \times 12.5 \mathrm{~mm}$ and $1.5 \mathrm{~mm}$ thick resin block was 3D-printed and embedded in a condensation silicon impression material (Genie putty, Sultan Healthcare, Hackensack, NJ) to create a mold for the wax pattern. Modelling wax (GEO Classic, gray opaque; Renfert Dental Corp, Hilzingen, Germany) was poured into the silicon mold; the patterns' thickness and dimensions were measured using a digital caliper (Dentagauge 2, Erskine Dental, USA) sensitive to $0.01 \mathrm{~mm}$. An 8-gauge and $6 \mathrm{~mm}$ long wax (Emporium, Florida USA) was used to connect the wax patterns to the investment ring (200g, IPS e.max Investment Ring System; IvoclarVivadent, Schaan, Liechtenstein). The patterns were 3 $\mathrm{mm}$ apart and a space of $10 \mathrm{~mm}$ was maintained between the ring and the patterns laterally and upward. (Freeman, Ohio, USA) (Figure 2). 


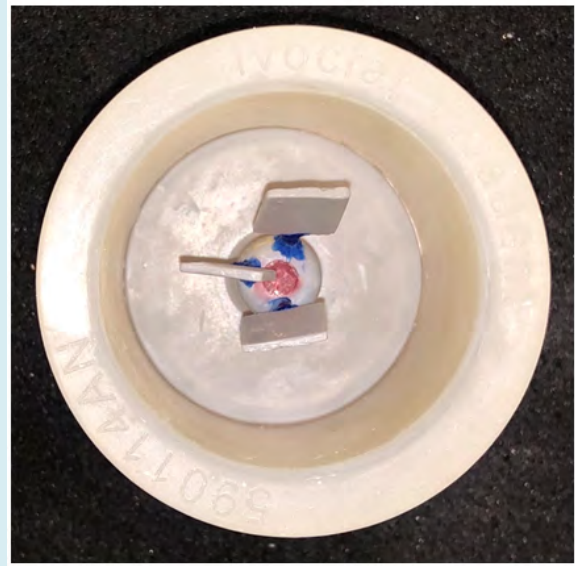

Figure 2: Three wax patterns attached to the silicon ring.

The investment was performed according to the manufacturer's recommendations. The IPS.emax specimens were allowed to set for 45 minutes and the Celtra Press specimens for 30 minutes. Thereafter, the invested material was placed face down in a pre-heated furnace (Ney 3-130 A Venturi Air Furnace, Esswein, Canada) for 1 hour at $850^{\circ} \mathrm{C}$. The ceramic ingot was positioned over the sprue access of the investment ring set and then fired $\left(865^{\circ} \mathrm{C}\right.$ for $\left.30 \mathrm{~min}\right)$ using a heat-pressing ceramic oven (ProgramatEP5000, IvoclarVivadent, Schaan, Liechtenstein). The investment compound was cut with a large carbide disc and the investment was removed with an aluminum oxide sand blaster (Renfert, Hilzingen, Germany) with $50 \mu \mathrm{m}$ glass beads at 3 bars pressure. The sprues were cut into the disc specimens with a diamond separating desk bur (Neytech, Dentsply, Germany) and all discs were measured again with the same digital caliper for a uniform thickness of $1.5 \mathrm{~mm}$ (Figure 3).

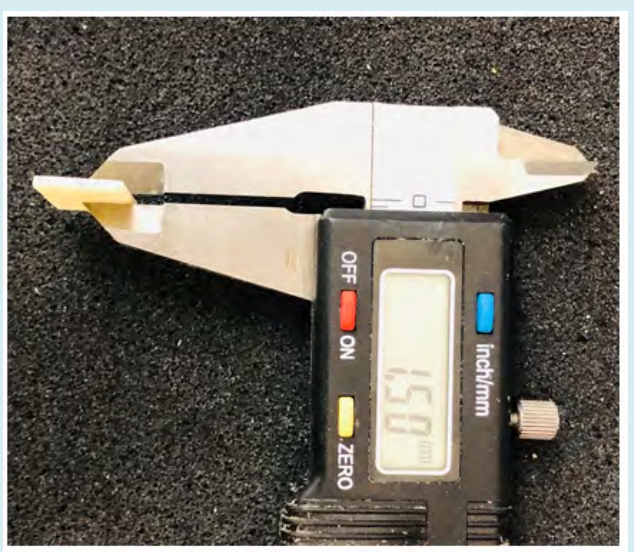

Figure 3: Ceramic disc thickness with digital caliper.

Polishing was performed using the manufacturer's recommended porcelain polishing burs on a bench vacuum
(Bessey, Stuttgart, Germany) to ensure secure seating of the samples. The Celtra Press Starter Kit (Dentsply, Germany) was used for Group 1 and Dialite Extra-Oral Polishing (Brasseler, Ventura, CA) for Group 2. Polishing was performed manually by one operator using the lab's micro-motor handpiece (Ultimate XI-K, NSK, Japan), and the specimens were polished for $30 \mathrm{sec}$, with 12 strokes and a consistent pressure. Polishing burs were used at a polishing speed of $15000 \mathrm{rpm}$ followed by high gloss polishing at 5000 rpm. Glazing in Group 1 was performed with CeltraTM Universal Glaze (Dentsply, Benshiem, Germany) at a 2-min firing cycle (500/932), while in Group 2, IPS Ivoclar Glaze (IvoclarVivadent, Schaan, Liechtenstein) was used at a 6-min firing cycle (403/769). For the combined polished and glazed groups, the samples were polished first and then glazed according to the manufacturer's recommendation, after which all of the samples were fired in a furnace (Programat P300, Ivoclar Vivadent).

All samples were randomized via a random sequence generator from www.random.org and each group was divided into four subgroups: No Treatment (A); Polished (B); Glazed (C); Polished and Glazed (D). Flexural strength was tested with a compressive load applied by a flat circular tungsten piston (Figure 4). Measurements were calculated by a universal testing machine (Instron) and the maximum load-to-failure (MPa) was recorded.

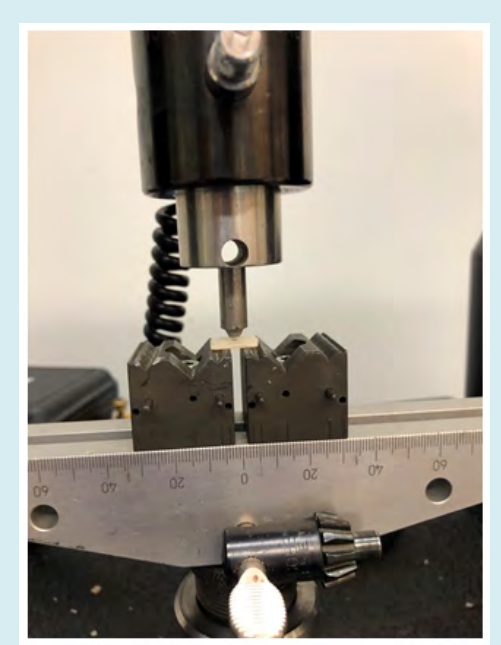

Figure 4: Universal testing machine (Model 5566; Instron, Canton).

Descriptive statistics (mean, standard deviation, minimum, and maximum) were calculated for each combination of material and surface treatment. Statistical significance was assessed via two-way ANOVA, with Tukey's HSD used in post-hoc comparisons of the surface treatments. Levene's test was used to evaluate the assumption of homogeneity of variance, and the Shapiro-Wilk test to 
evaluate the assumption of normality. The significance level was set at $\alpha=0.05$. SPSS v. 26 was used in the analysis.

\section{Results}

The descriptive statistics for all groups are shown in Table 1. The combination of IPS e.max with the polished and glazed surface treatment resulted in the highest mean flexural strength $(374.22 \pm 87.35 \mathrm{MPa})$, while the combination of Celtra Press with glazing only produced the lowest mean flexural strength $(164.10 \pm 51.97 \mathrm{MPa})$. In the two-way ANOVA, IPS e.max exhibited statistically significantly greater flexural strength than Celtra Press $(\mathrm{p}<$ 0.001 ), and the difference between the surface treatments was also statistically significant ( $p=0.007)$. In post-hoc comparisons of the surface treatments, Tukey's HSD test revealed that Group D had significantly greater flexural strength than Group A ( $p=0.010)$. Levene's test showed no significant evidence that the assumption of homogeneity of variances was violated $(\mathrm{p}=0.103)$, nor was there significant evidence of non-normality based on the Shapiro-Wilk test ( $p$ $=0.859$ ). Figure 5 presents box plots of the flexural strength results by material and surface treatment.

\begin{tabular}{|c|c|c|c|c|c|}
\hline Material & Surface treatment & Mean & SD & Minimum & Maximum \\
\hline Celtra Press & No Treatment & 187.56 & 57.61 & 132.62 & 288.28 \\
\hline IPS e.max & No Treatment & 269 & 83.18 & 132.29 & 371.92 \\
\hline Celtra Press & Polished & 205.06 & 30.1 & 169.95 & 242.33 \\
\hline IPS e.max & Polished & 363.7 & 48.57 & 273.41 & 434.57 \\
\hline Celtra Press & Glazed & 164.1 & 51.97 & 101.43 & 226.39 \\
\hline IPS e.max & Glazed & 322.22 & 88.61 & 206.91 & 444.31 \\
\hline Celtra Press & Polished and Glazed & 240.78 & 78.69 & 155.51 & 352.32 \\
\hline IPS e.max & Polished and Glazed & 374.22 & 87.35 & 237.26 & 458.19 \\
\hline
\end{tabular}

Table 1: Descriptive statistics of flexural strength (MPa) by material and surface treatment ( $\mathrm{n}=8$ per group).

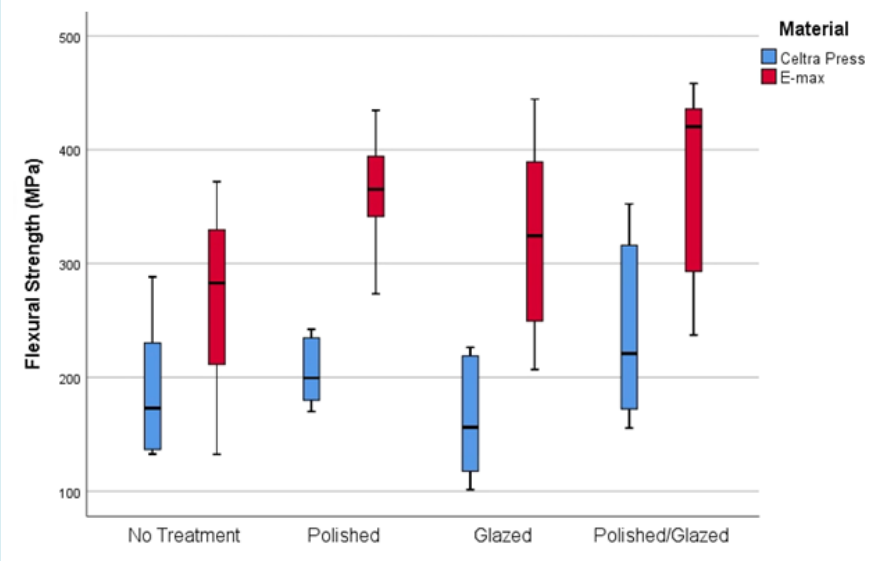

Figure 5: Box plots of the flexural strength results by material and surface treatment.

\section{Discussion}

This in-vitro study was designed to compare the flexural strength of heat-pressed zirconia-reinforced lithium silicate (CeltraPress, Dentsply Sirona, York, PA) with that of lithium disilicate ceramic (IPS e.max Press, Ivoclar Vivadent, Schaan, Liechtenstein) and to determine the surface treatments that could improve their flexural strength. The findings indicated that IPS e.max Press demonstrated statistically significantly higher biaxial flexural strength than Celtra Press, and a statistically significant difference was found also between the surface treatments, in that the flexural strength of the polished and glazed specimens was significantly higher than that of the untreated group.

All ceramic materials' clinical indications can be formulated using the ISO 6872:2015 specifications [15]. According to the results of this study, IPS e.max Press 


\section{Open Access Journal of Dental Sciences}

demonstrated a mean flexural strength between 269$374 \mathrm{MPa}$, which implies that it can be used for single-unit anterior or posterior restorations and three-unit anterior prostheses. Celtra Press's mean flexural strength tended to be less than $240 \mathrm{MPa}$, which indicates that it can be utilized only for single-unit anterior and posterior restorations.

According to the manufacturer, Celtra Press's mean flexural strength reaches $500 \mathrm{MPa}$ for glazed and polished specimens without thermocycling aging [14]. In contrast, the mean bi-axial flexural strength of polished and glazed Celtra Press samples in this study reached only $240.78 \mathrm{MPa}$. This is consistent with the results of Alakkad, et al. [16], in which polished and glazed Celtra Duo specimens' mean biaxial flexural strength was $238.03 \mathrm{MPa}$. Another study that compared the bi-axial flexural strength of IPS e.max CAD and Celtra Duo showed that the mean value of glazed and polished Celtra Duo specimens was 177.32 MPa [17]. Differences in the two formulations' composition and processing can account for the heat-pressed specimens' slightly greater flexural resistance compared to their CAD counterparts.

A number of studies has investigated the effect of $\mathrm{ZrO}_{2}$ on lithium disilicate ceramics' crystallization process [1820]. IPS e.max mechanically efficient is mainly attributed to the presence of elongated disilicate crystals that are layered and interlocked firmly, and the mismatch in the coefficient of thermal expansion between the glassy matrix and lithium disilicate crystals, which induces a tangential, compressive stress around the crystals. The addition of zirconia particles causes the crystals to align randomly in a non-parallel direction, and thus allows cracks to propagate easily [2123]. Incorporation of $\mathrm{ZrO}_{2}$ also prevented the complete transformation of lithium metasilicate, which has inferior mechanical properties, to lithium disilicate [22]. Increasing the $\mathrm{ZrO}_{2}$ content up to $10 \mathrm{wt} \%$ has been found to reduce the length of lithium disilicate's crystals, which could also affect the interlocking process [21]. These factors may explain why Celtra Press exhibited lower flexural strength in the current study.

With respect to the surface treatments, various studies have evaluated the strengthening effect attributable to the process used to polish ceramic materials $[24,25]$. However, polishing must be performed gently to avoid generating stress that will affect the material's structure adversely [26]. Ahmad, et al. [27] stated that the heat generated during polishing may cause thermal expansion on the material's surface that creates a residual tension. Our study showed that polishing affected both materials' flexural resistance positively. A standardized polishing method recommended by the manufacturer was used, which determined the amount of time the material was polished, as well as the mean polishing load. Hence, this study's findings can be compared only to similar polishing methods.

Although the difference between the glazed (Group B) and untreated specimens (Group A) was not statistically significant, it must be noted that the Celtra Press glazed group had a lower mean flexural strength $(164.10 \pm 51.97 \mathrm{MPa})$ than did the untreated group $(187.56 \pm 57.61 \mathrm{MPa})$. This finding is consistent with Yener, et al. [28], who reported that glazing reduced all specimens' flexural strength substantially. Another study reported that Ceramill, Cercon, and Zirkonzahn zirconia's biaxial flexural strength decreased following glazing as well [29]. One possible explanation for this is the mismatch in the coefficient of thermal expansion during the fire glazing that may create residual stress and compromise the flexural strength. This in-vitro study's primary limitation was that it did not consider factors encountered in the oral cavity. Thermocycling was also not performed, which according to the manufacturer, would have a positive effect on Celtra Duo's flexural strength.

\section{Conclusion}

Within the limitations of this study, it can be concluded that IPS e.max Press shows higher bi-axial flexural strength than Celtra Press. In addition, the combination of polished and glazed surface treatment resulted in greater flexural strength than that of the untreated group.

\section{References}

1. Schley JS, Heussen N, Reich S, Fischer J, Haselhuhn K, et al. (2010) Survival probability of zirconia-based fixed dental prostheses up to $5 \mathrm{yr}$ : A systematic review of the literature. Eur J Oral Sci 118(5): 443-450.

2. Asai T, Kazama R, Fukushima M, Okiji T (2010) Effect of overglazed and polished surface finishes on the compressive fracture strength of machinable ceramic materials. Dent Mater J 29(6): 661-667.

3. Conrad HJ, Seong WJ, Pesun IJ (2007) Current ceramic materials and systems with clinical recommendations: a systematic review. J Prosthet Dent 98(5): 389-404.

4. McLaren EA, Cao PT (2009) Ceramics in dentistry-Part I: Classes of materials. Inside Dentistry 5(9): 94-103.

5. Rekow ED, Silva NR, Coelho PG, Zhang Y, Guess P, et al. (2011) Performance of dental ceramics: Challenges for improvements. J Dent Res 90(8): 937-952.

6. Guess PC, Schultheis S, Bonfante EA, Coelho PG, Ferencz JL, et al. (2011) All-ceramic systems: Laboratory and clinical performance. Dent Clin North Am 55(2): 333352. 
7. Press C (2018) Zirconia-Reinforced Lithium Silicate ZLS In: Dentsplysirona.

8. Holand W, Apel E, vant Hoen C, Rheinberger V (2006) Studies of crystal phase formation in the early stage crystallization of lithium disilicate glass-ceramics. J Non Cryst Solids 352(38-39): 4041-4050.

9. Holand W, Schweiger M, Frank M, Rheinberger V (2000) A comparison of the microstructure and properties of the IPS Empress 2 and the IPS Empress glass-ceramics. J Biomed Mater Res 53(4): 297-303.

10. Kang SH, Chang J, Son HH (2013) Flexural strength and microstructure of two lithium disilicate glass ceramics for CAD/CAM restoration in the dental clinic. Restor Dent Endod 38(3): 134-140.

11. Al Wahadni A, Muir Martin D (1998) Glazing and finishing dental porcelain: A literature review. J Canadian Dent Assoc 64(8): 580-583.

12. Steiner R, Beier US, Heiss Kisielewsky I, Englemeier R, Dumfahrt H, et al. (2015) Adjusting dental ceramics: An in vitro evaluation of the ability of various ceramic polishing kits to mimic glazed dental ceramic surface. J Prosthet Dent 113(6): 616-622.

13. Levy H (1987) Effect of laboratory finishing technics and the mechanical properties of dental ceramic. L'Information Dentaire 69(12): 1039-1045.

14. Rosenstiel SF, Baiker MA, Johnston WM (1989) Comparison of glazed and polished dental porcelain. Int J Prosthodont 2(6): 524-529.

15. Dentistry: Ceramic materials. (2015) International Organization for Standardization Geneve.

16. Alakkad L (2018) Flexural strength of various CAD/CAM ceramic materials (Masters Thesis). Tufts University School of Dental Medicine.

17. Badawy RE S, El Mowafy O, Tam L (2016) Flexural properties of chairside CAD/CAM materials. Dent Med Prob 53(2): 230-235.

18. Thieme K, Rüssel C (2015) Nucleation and growth kinetics and phase analysis in zirconia-containing lithium disilicate glass. J Mat Science 50: 1488-1499.

19. Apel E, van't Hoen C, Rheinberger V, Holand W et al. (2007) Influence of $\mathrm{ZrO}_{2}$ on the crystallization and properties of lithium disilicate glass-ceramics derived from a multi-component system. J Europ Ceramic Soc 27(2-3): 1571-1577.
20. Krüger S, Deubener J, Ritzberger C, Holand W (2013) Nucleation kinetics of lithium metasilicate in $\mathrm{ZrO}_{2}-$ bearing lithium disilicate glasses for dental application. International Journal of Applied Glass Science 4(1): 9-19.

21. Hallmann L, Ulmer P, Kern M (2018) Effect of microstructure on the mechanical properties of lithium disilicate glass-ceramics. J Mech Behav Biomed Mater 82: 355-370.

22. Huang X, Zheng X, Zhao G, Zhong B, Zhang X, et al. (2014) Microstructure and mechanical properties of zirconiatoughened lithium disilicate glass-ceramic composites. Materials Chemistry and Physics. 143(2): 845-852.

23. Bischoff C, Eckert H, Apel E, Rheinberger VM, Holand $W$ (2011) Phase evolution in lithium disilicate glassceramics based on non-stoichiometric compositions of a multi-component system: Structural studies by $29 \mathrm{Si}$ single and double resonance solid state NMR. Phys Chem Chem Phys 13(10): 4540-4551.

24. de Jager N, Feilzer AJ, Davidson CL (2000) The influence of surface roughness on porcelain strength. Dent Mat 16(6): 381-388.

25. Brackett SE, Leary JM, Turner KA, Jordan RD (1989) An evaluation of porcelain strength and the effect of surface treatment. J Prosthet Dent 61(4): 446-451.

26. Giordano RA, Campbell S, Pober R (1994) Flexural strength of feldspathic porcelain treated with ion exchange, overglaze, and polishing. J Prosthet Dent 71(5): 468-472.

27. Ahmad R, Wu B, Morgano S (2001) Polishing mechanism and its effect on the mechanical properties of ceramic restorations-A review of the literature. Annals of Dentistry, University of Malaya 8(1): 57-61.

28. Yener ES, Ozcan M, Kazazoglu E (2011) The effect of glazing on the biaxial flexural strength of different zirconia core materials. Acta Odontol Latinoam 24(2): 133-140.

29. Yener ES, Ozcan M, Kazazoglu E (2015) A comparative study of biaxial flexural strength and Vickers microhardness of different zirconia materials: Effect of glazing and thermal cycling. Brazilian Dental Science 18(2): 19-30. 